\title{
Local FK506 implants in non-human primates to prevent early acute rejection in vascularized composite allografts
}

\begin{abstract}
Alexandre G. Lellouch ${ }^{1,2,3} \wedge$, Corentin B. Taveau ${ }^{1,2,3}$, Alec R. Andrews ${ }^{1,2}$, Joseph Molde ${ }^{4}$ Zhi Yang Ng ${ }^{1,2,5} \wedge$, Philipp Tratnig-Frankl ${ }^{1,2,6}$, Ivy A. Rosales ${ }^{7}$, Marion Goutard ${ }^{1,2,3}$, Elise Lupon ${ }^{1,2}$, Laurent A. Lantieri ${ }^{3}$, Robert B. Colvin ${ }^{7}$, Mark A. Randolph ${ }^{1,2}$, Joachim Kohn ${ }^{4}$, Curtis L. Cetrulo Jr ${ }^{1,2,8}$

${ }^{1}$ Division of Plastic and Reconstructive Surgery, Massachusetts General Hospital, Harvard Medical School, Boston, MA, USA; ${ }^{2}$ Vascularized Composite Allotransplantation Laboratory, Center for Transplantation Sciences, Massachusetts General Hospital, Harvard Medical School, Boston, MA, USA; ${ }^{3}$ Service de Chirurgie Plastique, Hôpital Européen Georges Pompidou, Assistance Publique-Hôpitaux de Paris (APHP), Université Paris Descartes, Paris, France; ${ }^{4}$ Department of Life Sciences, The New Jersey Center for Biomaterials, Rutgers-The State University of New Jersey, Piscataway, NJ, USA; ${ }^{5}$ Plastic Surgery, School of Surgery, Oxford, UK; ${ }^{6}$ Department of Plastic, Reconstructive and Aesthetic Surgery, Vienna General Hospital, Medical University of Vienna, Vienna, Austria; ${ }^{7}$ MGH Transplant Center, Massachusetts General Hospital, Harvard Medical School, Boston, MA, USA; ${ }^{8}$ Shriners Hospital for Children, Boston, MA, USA

Contributions: (I) Conception and design: AG Lellouch, ZY Ng, J Kohn, CL Cetrulo Jr; (II) Administrative support: AG Lellouch, AR Andrews, MA Randolph, ZY Ng; (III) Provision of study materials or patients: J Molde, J Kohn; (IV) Collection and assembly of data: AG Lellouch, CB Taveau, AR Andrews, J Molde, ZY Ng, PT Tratnig-Frankl, M Goutard, E Lupon; (V) Data analysis and interpretation: All authors; (VI) Manuscript writing: All authors; (VII) Final approval of manuscript: All authors.

Correspondence to: Curtis L. Cetrulo Jr, MD, FACS, FAAP. Division of Plastic and Reconstructive Surgery, Massachusetts General Hospital, 15 Parkman Street, Wang 435, Boston, MA 02114, USA. Email: ccetrulo@mgh.harvard.edu.
\end{abstract}

Background: Previous vascularized composite allograft (VCA) studies from our laboratory have shown that topical FK506 delivery in non-human primates (NHPs) was limited by inadequate dermal penetration and rejection persisted. Herein, we report the first utilization of FK506 via subcutaneously implanted discs to mitigate VCA rejection in NHPs.

Methods: Full major histocompatibility complex (MHC)-mismatched NHP pairs underwent partial-face VCA and FK506 disc implantation along the suture line. All allotransplants were maintained post-operatively for two months on the FK506 discs, methylprednisolone, mycophenolate mofetil, and supplemented with intramuscular FK506 if necessary. Group $1(n=4)$ was used for optimization of the implant, while Group $2(n=3)$ underwent delayed bone marrow transplantation (DBMT) after two months. VCA skin biopsies and peripheral blood samples were obtained for serial assessment of rejection and mixed chimerism by histopathology and flow cytometry respectively.

Results: In Group 1, two technical failures occurred. Of the remaining two NHPs, one developed supratherapeutic levels of FK506 (50-120 ng/mL) and had to be euthanized on postoperative day (POD) 12. Reformulation of the implant resulted in stable FK506 levels (20-30 ng/mL) up to POD12 when further intramuscular (IM) FK506 injections were necessitated. In Group 2, two NHPs survived to undergo conditioning and one successfully developed chimerism at 2-3 weeks post-DBMT (96-97\% granulocytes and $7-11 \%$ lymphocytes of recipient-origin). However, all three NHPs had to be terminated from study at POD64, 77 and 86 due to underlying post-transplant lymphoproliferative disorder. All VCAs remained rejection-free up to study endpoint otherwise.

Conclusions: This study shows preliminary results of local FK506 implants in potentially mitigating VCA acute rejection for tolerance protocols based on mixed chimerism approach.

^ ORCID: Alexandre G. Lellouch, 0000-0001-8191-8662; Zhi Yang Ng, 0000-0003-0538-3066. 
Keywords: Immunosuppression; vascularized composite allograft (VCA); tacrolimus

Submitted Jan 20, 2021. Accepted for publication Mar 28, 2021.

doi: $10.21037 /$ atm-21-313

View this article at: http://dx.doi.org/10.21037/atm-21-313

\section{Introduction}

It has been more than 20 years since the first successful hand transplant (1) and the utility of vascularized composite allografts (VCAs) remains plagued by the same fundamental problem, lifelong systemic immunosuppression and the attendant sequelae of life-threatening complications (2-4). Compared to solid organ transplantation (SOT), VCAs differ in the composition of the transplanted tissues including the skin that presents a higher antigenic load potentially requiring even more immunosuppression $(5,6)$. Based on the latest report of the International Registry on Hand and Composite Tissue Allotransplantation in 2017, patients compliant with triple immunosuppressive regimens (i.e., calcineurin inhibitor, steroids, mycophenolate mofetil (MMF) were able to maintain and prevent early loss of the VCA. However, acute rejection of the VCA occurs in up to $90 \%$ of patients, over the first year post transplantation, with almost $60 \%$ developing multiple episodes (2). The exteriorized skin component of VCAs could present an opportunity for earlier detection and prompt treatment of rejection with topical immunosuppression. The clinical experience in VCA however, has more or less confirmed that while topicals may help reverse rejection episodes, further titration and boluses of systemic immunosuppression (typically steroids) were often necessary $(7,8)$. Indeed, our laboratory has recently reported that topical FK506 did not allow the reduction of systemic maintenance immunosuppression in VCAs in a non-human primate (NHP) model and could only penetrate into the epidermis (9). Additionally, we have demonstrated through in vitro analysis of NHP VCA skin biopsies that by week 2, skin leukocyte populations (CD4+, CD8+) within the dermis have undergone near-complete $(>90 \%)$ turnover from donor (VCA) to recipient-origin (10). Nonetheless, VCA rejection episodes were unavoidable and rendered subsequent immune tolerance protocol attempts through mixed chimerism using bone marrow transplantation impossible (10).

Inducing a state of immunologic tolerance to allotransplants in the absence of immunosuppression would greatly expand the clinical application of VCA. Mixed chimerism, in which both donor- and recipient-derived lymphohematopoietic elements co-exist in the recipient (11), may negate the spectre of chronic rejection, and is a proven strategy to enable immunosuppression withdrawal as demonstrated in clinical trials on renal transplantation (12). Many reported strategies to induce mixed chimerism rely on 1-2 days of recipient preconditioning, including viable donor cell infusions, which would not be logistically possible for VCAs from cadaveric donors. Using a delayed tolerance induction protocol (DTIP), whereby the donor cells are temporarily stored for infusion 1-2 months after VCA, could be one approach if acute rejection was mitigated during the delay period.

A variety of research efforts are underway to develop drug delivery systems (DDS) that may mitigate rejection in VCA $(13,14)$. All previous DDS studies have been limited to small animal models that arguably, have limited translational potential, especially in the context of VCA (15), except for a recent porcine study by Fries et al. (16). Those authors showed that tacrolimus-loaded hydrogel implanted into the allograft delayed the onset of Grade IV acute rejection in porcine forelimb VCAs. In this study, we describe our experience in replacing our previous topical FK506-TyroSpheres (in the form of a gel dressing acting mainly on the epidermis) (9) with subcutaneously implanted discs for direct and sustained release into VCA dermis. The primary and secondary aims of our study are thus mitigation of acute rejection, and the successful development of delayed mixed chimerism respectively. The attainment of the former is expected to enable the latter to be achieved. We present the following article in accordance the ARRIVE reporting checklist (available at http://dx.doi.org/10.21037/atm-21-313).

\section{Methods}

All experiments were performed in accordance with the NIH's Guide for the Care and Use of Laboratory Animals with approval from the IACUCs of Rutgers University and Massachusetts General Hospital (2015N000146). 


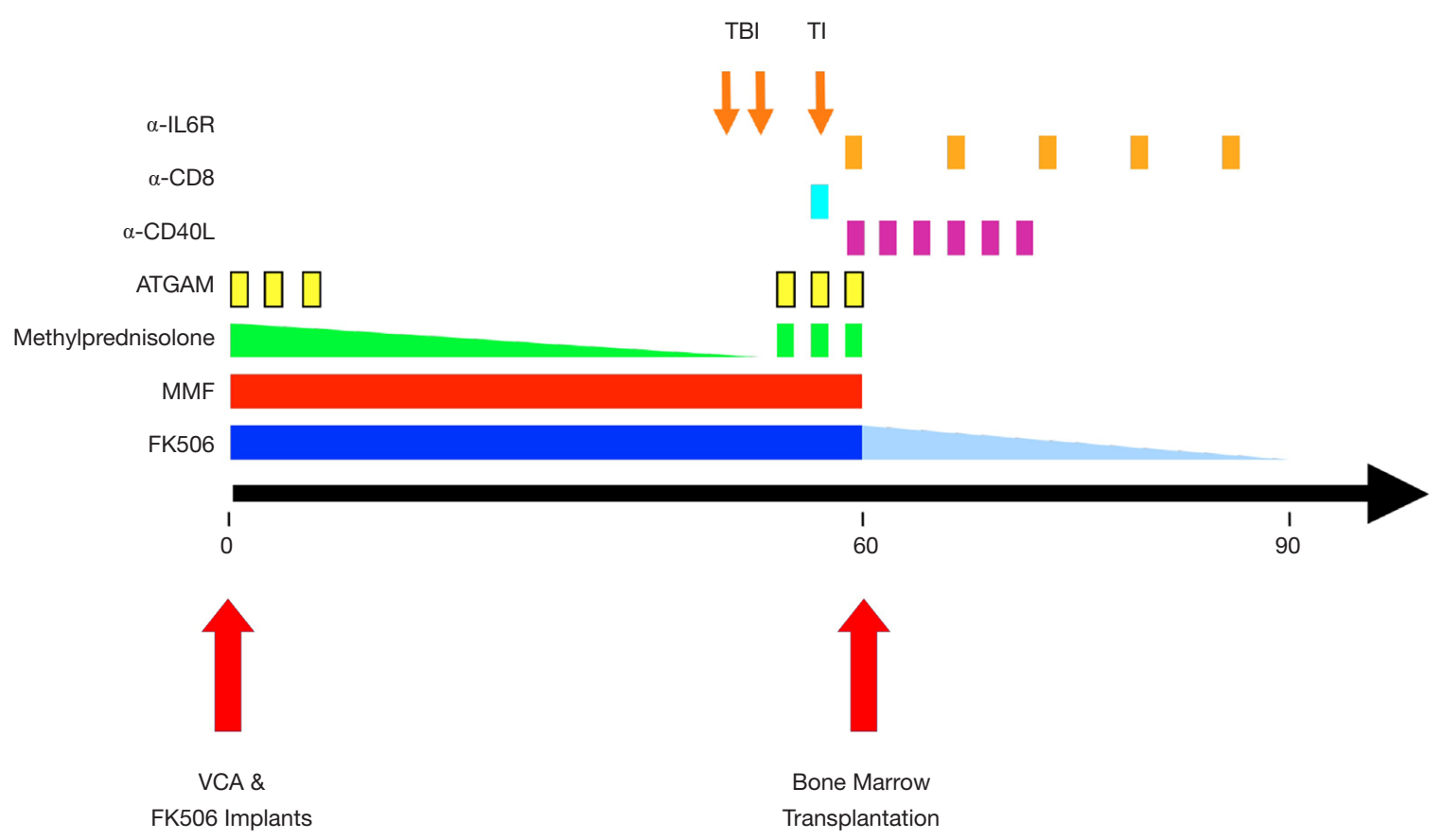

Figure 1 Delayed tolerance induction protocol for VCA. The sequence and timing of the protocol interventions are depicted relative to the timing of VCA and DBMT. The VCAs were maintained with conventional triple-drug immunosuppression for 60 days, followed by DBMT using a nonmyeloablative conditioning regimen including total body irradiation (TBI, 150 cGy, on POD 54 and 55), thymic irradiation (TI, 700 cGy on POD 59), ATGAM (IV $50 \mathrm{mg} / \mathrm{kg}$ on POD 58, 59 and 60), methylprednisolone (IV $5 \mathrm{mg}$ on POD 58, 59 and 60) and anti-CD8 $\mathrm{mAb}$ (cM-T807, Centocor, Inc., Horsham, PA; $5 \mathrm{mg} / \mathrm{kg}$ IV on POD 59). After DBMT, the recipients were treated with short courses of anti-CD40L (h5C8; $20 \mathrm{mg} / \mathrm{kg}$ IV on POD 60 and 62, and $10 \mathrm{mg} / \mathrm{kg}$ on POD 65, 67, 69, and 72) and anti-IL-6 receptor mAb (tocilizumab, ACTEMRA $^{\circledR}$; Genentech, San Francisco, CA; $10 \mathrm{mg} / \mathrm{kg}$ IV on POD 0, 67, 74, 81 and 88). After this, no further immunosuppression was given. ATGAM, equine anti-thymocyte globulin; DBMT, delayed bone marrow transplantation; mAb, monoclonal antibody; POD, postoperative day; TBI, total body irradiation; TI, thymic irradiation; VCA, vascularized composite allograft.

\section{Experimental design}

Adult, male cynomolgus monkeys (Macaca fasicularis) weighing 5 to $10 \mathrm{~kg}$ were chosen for this study (Charles River Primates, Wilmington, MA). Transplant pairs were selected for compatible $\mathrm{ABO}$ blood types and full major histocompatibility complex (MHC)-mismatching $(17,18)$ before undergoing heterotopic, partial face VCA (19).

In Group 1, four NHPs were used to optimize the pharmacokinetics of FK506 delivery. In Group 2, three NHPs received the optimized FK506 implants and were maintained on supplemental FK506 as necessary along with standard methylprednisolone, and MMF. Animals in the latter group underwent the DTIP involving preoperative thymic and total body irradiation along with other conditioning agents, as described previously (10) and detailed in Figure 1, prior to receiving delayed bone marrow transplantation (DBMT) at 60 days.

\section{Optimization of local FK506 implant design}

Details of the in vitro and in vivo testing and manufacturing of the FK506 implants in Sprague-Dawley rats are beyond the scope of the current study and have been reported elsewhere (20). Essentially, scale-up iterations of the FK506 implants were cycled through Group 1 before arriving at the final formulation for Group 2, and all were distributed evenly along the suture line between donor (VCA) and host skin.

The initial iteration of FK506 implants (15 wt\% film measuring $10 \mathrm{~mm} \times 10 \mathrm{~mm}$ each) in Group 1 was based on the release profile, tissue distribution and standard intravenous (IV) or intramuscular (IM) FK506 dosing protocol of $0.1 \mathrm{mg} / \mathrm{kg} /$ day. Based on the timeline of the DTIP where immunosuppression lasts up to 90 days 

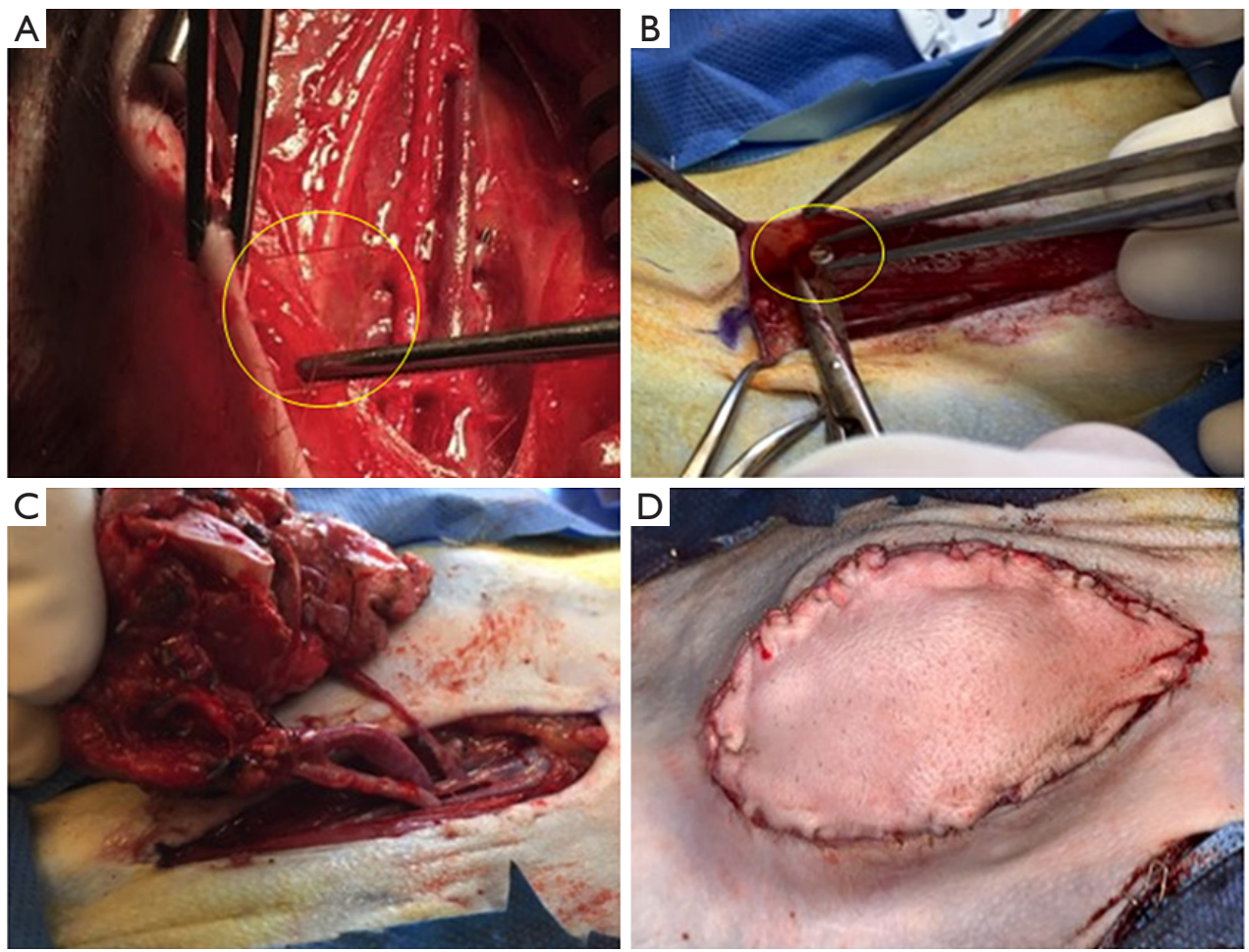

Figure 2 Development of local FK506 implant technology: (A) initial, unsuccessful iterations consisting of $15 \%$ drug-loaded devices measuring $10 \mathrm{~mm} \times 10 \mathrm{~mm}$ each (10× film and $1 \times$ laminated versions), and (B) final, successful iteration consisting of $4 \mathrm{~mm}$ diameter circular implants loaded with $30 \mathrm{wt} \%$ tacrolimus and $1 \mathrm{wt} \%$ vitamin $\mathrm{E}$ (stabilizer to prevent degradation following sterilization and storage) totaling $3 \mathrm{~cm}^{2}$ (C) placed along the suture line and (D) final appearance on skin closure. Implants circled in yellow to aid visualization.

(60 days maintenance and 30 days bridging post-DBMT), total dosages were:

* IV/IM FK506 = $0.1 \mathrm{mg} / \mathrm{kg} /$ day $\times 10 \mathrm{~kg} / \mathrm{NHP} \times$ 90 days $=90 \mathrm{mg} / \mathrm{NHP}$.

To provide the equivalent dose via FK506 implants,

- Implant FK506 = $0.15 \mathrm{mg} / \mathrm{mg}$ film $\times 60 \mathrm{mg}$ film $/$ implant $\times 10$ films/NHP $=90 \mathrm{mg} / \mathrm{NHP}$.

Therefore, ten $15 \%$ FK506-loaded films were sterilized intra-operatively with povidone-iodine before implantation (in the first two NHPS in Group 1) at least $20 \mathrm{~mm}$ apart around the transplant site (Figure $2 A, B, C, D$ ). The in vivo implant release profile (see Results section) was not consistent with that predicted by in vitro data, with a high initial release of FK506 followed by a second spike which required redesign. This was addressed (in the next two NHPs, M3 and M4, in Group 1) through lamination to seal in the drug and provide a diffusion barrier to prevent the initial burst release of FK506. Two implants were then placed in M3, while only one was used in M4; this was equivalent to a dosing of approximately 1.8 and $0.9 \mathrm{mg} / \mathrm{kg}$ respectively and models the daily IV/IM dose with the secondary goal of also extending local immunosuppression for as long as possible.

The final version of the FK506 implants in Group 2 was that of $4 \mathrm{~mm}$ circular implants (24 total) loaded with $30 \mathrm{wt} \%$ tacrolimus and $1 \mathrm{wt} \%$ vitamin $\mathrm{E}$ (stabilizer to prevent degradation following sterilization and storage) totaling $3 \mathrm{~cm}^{2}$ (based on in vivo drug release studies in rodents at Rutgers University). These implants were also sterilized pre-operatively by gamma irradiation to mirror actual clinical application. Such a design aimed to provide therapeutic levels of FK506 via controlled release over the first 7-10 days to combat acute rejection of the VCA with further IM supplementation thereafter as necessary. Despite the likely lower systemic levels, the presumed higher FK506 concentration within the soft tissue (based on rodent studies, no validated method in NHPs) surrounding the implants is hypothesized to provide adequate local immunosuppression throughout the lifetime of the implant to prevent acute rejection. Historical NHP subjects from 
our laboratory without FK506 implantation were previously reported and utilized as controls for this study (10).

\section{Immunological monitoring}

Post-transplantation, VCAs were monitored twice daily for the first 72 hours and once daily subsequently, or more frequently if requested. Protocol and for-cause VCA skin biopsies were performed using a standard $6 \mathrm{~mm}$ punch biopsy kit at approximately 30-day intervals. When rejection was suspected clinically (i.e., increased erythema, swelling, ulceration etc.), treatment was initiated with steroid bolus and gradual taper over 14 days to $1 \mathrm{mg}$ per day (same as maintenance). All VCA skin biopsies were fixed in formalin, stained with hematoxylin and eosin (H\&E), and subjected to pathologic analysis according to the 2007 Banff Working Classification for composite tissues $(21,22)$ by two pathologists (I.A.R and R.B.C) blinded to the study.

Peripheral blood chimerism was evaluated chronologically post-VCA by flow cytometry as described previously at approximately 2 -week intervals $(10,23)$. Systemic levels of FK506 were measured chronologically post-VCA with an Abbott ARCHITECT Immunoassay Analyzer.

\section{Statistical analysis}

Experience with large animal models for transplantation research over the past 30 years at $\mathrm{MGH}$ has demonstrated that three transplant recipients per experimental group are sufficient to provide statistical power for the detection of survival differences, and to achieve statistical significance in the comparison of categorically-scored histological specimens using non-parametric statistical tests (e.g., Mann-Whitney U) with an alpha risk level of $5 \%$ and a beta risk level of $80 \%$ (24).

\section{Results}

\section{Optimization of FK506 delivery}

In Group 1, M1 developed venous thrombosis of the VCA and lost the graft by POD 4. Systemic FK506 levels were measured up to POD 12 in M2 and ranged from 50-120 ng/mL. These levels were 2-6 fold higher than the therapeutic target range of $20-30 \mathrm{ng} / \mathrm{mL}$ (for NHPs) and were thought to have arisen from the combination of the induction dose of IM FK506 $(0.2 \mathrm{mg} / \mathrm{kg} \times 9 \mathrm{~kg}$ NHP $=1.8 \mathrm{mg}$ ) and high early release levels from FK506 implants that had been observed during the first few days from earlier in vitro studies in rats (not shown). The sustained and high release of FK506 past the first week, however, was cause for serious concern. Clinical assessment of M2 suggested potential tacrolimus toxicity including poor appetite, weight loss, and a slight tremor, resulting in removal of this animal from the study on POD 12.

The burst release of FK506 was addressed by lamination and reducing the number of implants (from ten) as described in Materials \& Methods, and implemented for the next two NHPs (M3, M4) in Group 1. M4 received two implants, developed venous thrombosis and lost the graft by POD 5. M3 received two implants, which reduced systemic levels of FK506 to within the desired therapeutic range up to POD 11 before additional IM dosing was required. M3 was then removed from study on POD 13 as it had reached the experimental end point of rejection (corresponding FK506 levels $<20 \mathrm{ng} / \mathrm{mL}$ ) (Figure 3).

In Group 2 NHPs (n=3), initial systemic FK506 levels at POD 3 were 60.0, 79.1 and $112.4 \mathrm{ng} / \mathrm{mL}$ and subsequent IM FK506 administration was stopped. In M5, FK506 levels fell rapidly to $26.6 \mathrm{ng} / \mathrm{mL}$ by POD 10, which necessitated reinstatement of IM FK506. In contrast, M6 did not require additional IM FK506 up to the point of DBMT (at POD 60). In this animal systemic FK506 levels had a second peak at week two $(100 \mathrm{ng} / \mathrm{mL})$ before gradually tapering to and maintaining just above the target range at $30-40 \mathrm{ng} / \mathrm{mL}$ from approximately POD 30 onwards. M7 had a similar course to M6 and only required additional IM FK506 from around POD 45 onwards when systemic FK506 levels fell below the target range (Figure 4).

\section{Successful mitigation of acute rejection}

Acute rejection episodes were avoided in all three Group 2 NHPs. However, routine CBC from M7 on POD 12 revealed a raised granulocyte count $\left(14.5 \times 10^{9} / \mathrm{L}\right)$ that manifested with a cutaneous rash on the VCA associated with pruritus. This was treated presumptively as rejection by titrating methylprednisolone back up to $40 \mathrm{mg} \mathrm{IV}$, and symptomatically with diphenhydramine $(50 \mathrm{mg} / \mathrm{kg} /$ day $)$ until clinical resolution was achieved. All Group 2 NHPs had VCA biopsies taken on POD 30 and POD 60 that returned negative for rejection (Figure 5).

\section{Detection of donor cells after DTIP but PTLD remained rampant}

All three NHPs in Group 2 successfully underwent the 


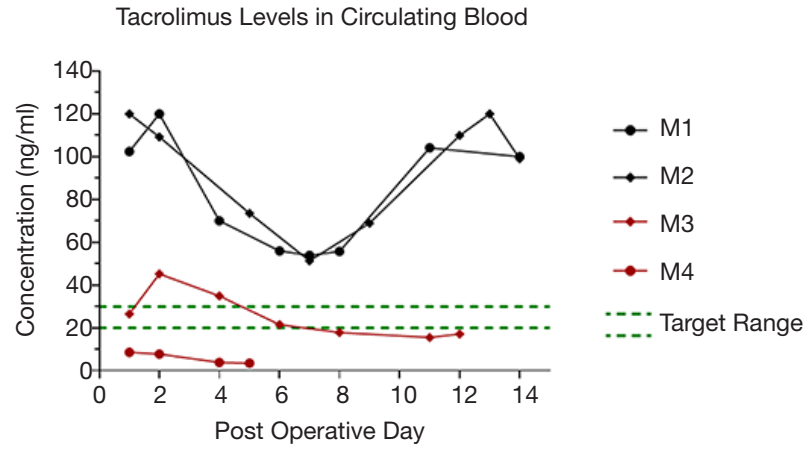

Figure 3 Systemic FK506 concentration comparison in Group 1: for the first iteration of NHP studies, M1 and M2, against the second iteration of NHP studies, M3 and M4. By using fewer laminated implants, systemic FK506 levels were brought to the target range for up to 11 days in vivo without requiring additional IM administration. NHP, non-human primate.

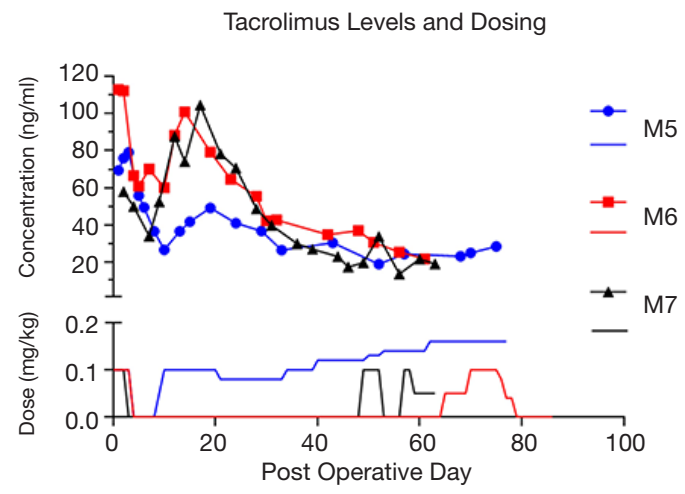

Figure 4 Systemic levels of FK506 (lines with symbols) in Group 2 following transplantation, and corresponding dosages of IM FK506 (solid lines). M5 required additional IM FK506 throughout the study whereas M6 did not, and M7 had a similar course until POD 45.

DTIP and received DBMT but developed progressive weight loss of between $30-35 \%$ of total body weight and worsening leukocytosis $\left(14 \times 10^{9} / \mathrm{L}-37.4 \times 10^{9} / \mathrm{L}\right)$ shortly after. Of note, M6 developed a period of impaired glucose tolerance with random blood sugar at $389 \mathrm{mg} / \mathrm{dL}$ on POD 29 while FK dosing was being maintained by only the subcutaneous implant. This was treated with a diabetogenic diet and insulin until POD 50. At a similar timepoint, M7 which also had FK dosing maintained solely by subcutaneous implant, developed lymphadenopathy of the right inguinal lymph node on POD 39. The lymph node was surgically excised and histology confirmed underlying post-transplant lymphoproliferative disorder (PTLD). Although the animal did go on to receive DBMT, it had lost $>20 \%$ of its total body weight by POD 64 and was also removed from study per veterinary recommendation. During this timeframe, all three NHPs were maintained with comparable systemic tacrolimus levels despite the varying clinical courses. Following DBMT, there was strong clinical suspicion for PTLD in M5 and M6, which was confirmed at the end of study on POD 77 and 86, respectively. PTLD were all likely of recipient-origin (Figure 6).

Final chimerism analysis showed a very low percentage of donor cells ( 2-5\%) at experimental endpoint in M5 and M7. M6 on the other hand successfully developed mixed chimerism (96-97\% granulocytes and 7-11\% lymphocytes of donor-origin) at 2-3 weeks post-DBMT (Figure 7).

\section{Discussion}

A plethora of drug delivery systems have been reported in small animal VCA models to mitigate acute rejection using various immunosuppressants including FK506, steroids, and rapamycin $(13,14)$. These include direct topical application $(25,26)$; intra-graft administration with various functionalities such as "on-demand" drug release in response to inflammation (27) and "on-cue" release upon ultrasound stimulation (28); and local biodegradable implants with sustained regional release (29). Most encouragingly, these reports have all had varying degrees of long-term (100-280 days) VCA survival, minimal systemic toxicity and related complications, and the potential for immunoregulation through chimerism and even increased levels of $\mathrm{T}_{\text {regs }}(30)$.

Our laboratory is focused on developing a similar approach to local immunosuppression but instead, we utilized a translational large animal model (NHPs). We have previously shown that topical FK506 was limited by inadequate dermal penetration in NHP when given alongside reduced levels of systemic immunosuppression (9). This outcome reflected the clinical experience in VCA, whereby topicals could only augment systemic therapy $(2,8)$. Hence, our current study was focused on directing local FK506 delivery to the dermis, similar to the reported experience in rats (29). Our main challenges, however, were to translate successes in small animal models for scale-up of in vivo testing in a pre-clinical NHP VCA model.

To the best of our knowledge, there has only been one prior large animal study on local immunosuppression. 

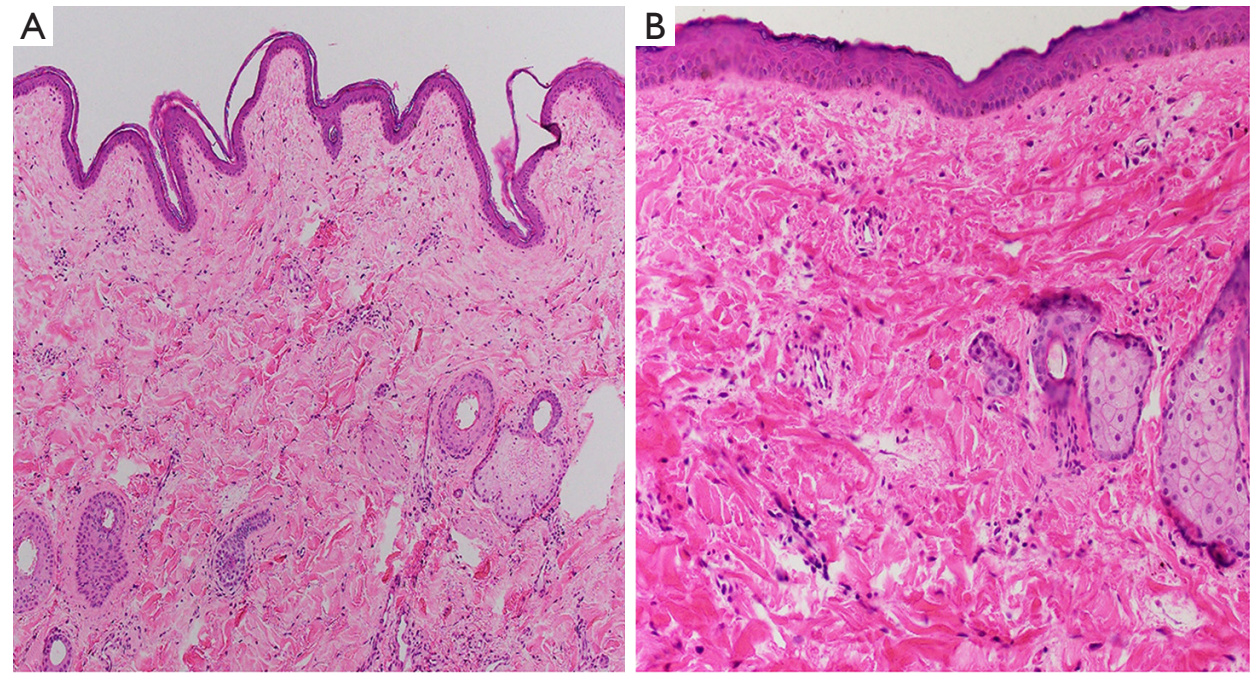

Figure 5 (A) M5 (H\&E, 100x) and (B) M7 (H\&E, 400x). The skin from the VCA shows no evidence of rejection at end of study. The epidermis is intact and shows no inflammation. VCA, vascularized composite allograft.
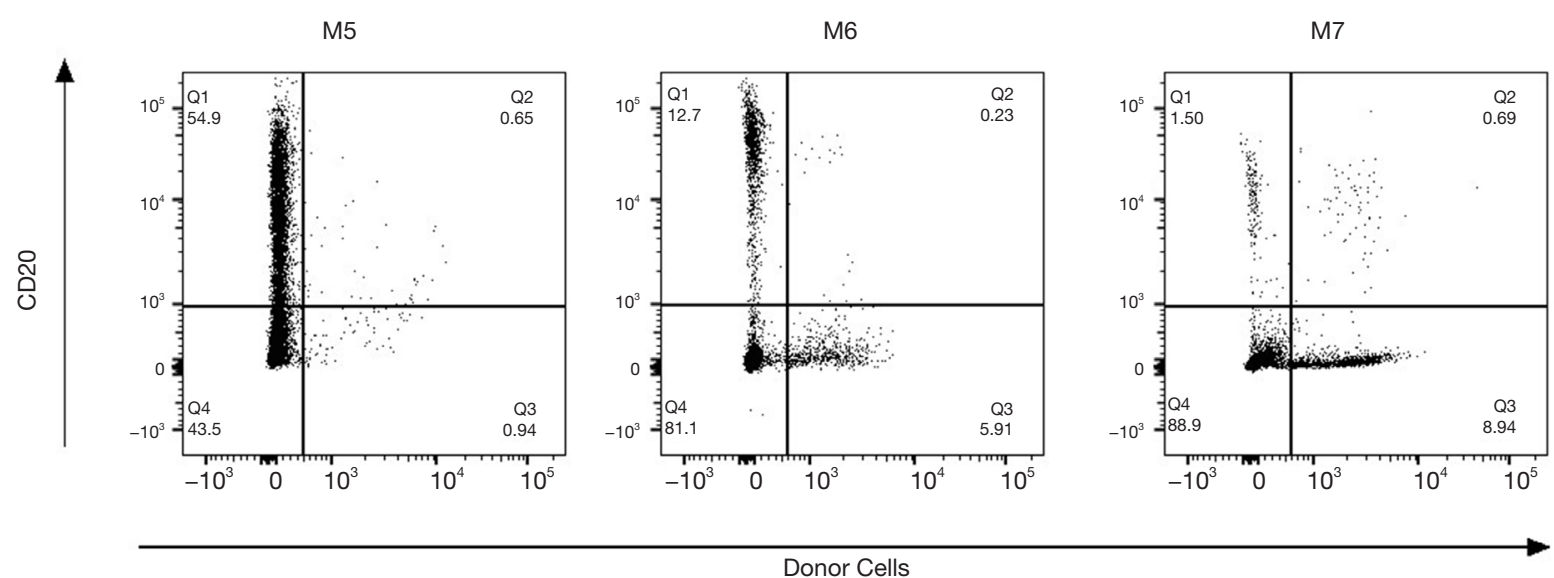

Figure 6 End-of-study whole blood data for each Group 2 recipient. PTLD likely of recipient origin in all recipients since there was minimal double positive staining in the CD20+ H38+ population (Q2). Majority of B-cells are recipient origin. PTLD, post-transplant lymphoproliferative disorder.

Fries et al. (16) investigated the use of high $(91 \mathrm{mg} / \mathrm{limb})$ and low-dose (49 mg/limb) FK506-loaded hydrogels alone, administered subdermally prior to skin closure in a porcine VCA model. Both experimental groups had similar early burst release of FK506 (range, 33.18-42.16 ng/mL and $11.91-35.62 \mathrm{ng} / \mathrm{mL}$ for high and low-dose groups respectively on POD 1) followed by a second spike around POD7-10. However, animals in the high-dose group had to be euthanized at POD 24, 30 and 42 due to failure to thrive, while low-dose group animals survived up to POD 56, 63, 91 and 93 before Grade IV rejection developed. Similarly,
NHP recipients in Group 2 of the current study developed a myriad of side effects, likely from the high dose of systemic FK506, including diabetes and the development of recipient-origin PTLD (in association with BMT). VCAs in the study by Fries et al. were performed across only a single haplotype mismatch rather than a full MHC-mismatch as in our current study. Furthermore, Fries' study was designed to consider graft survival as up to Grade IV rejection (i.e., necrosis). However, Grade I-II rejection developed from as early as POD 4 in both groups, an observation that is arguably of greater clinical importance, which underlies the 
M5

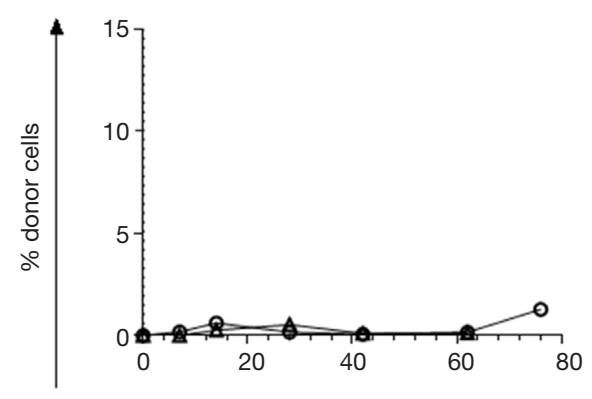

M6

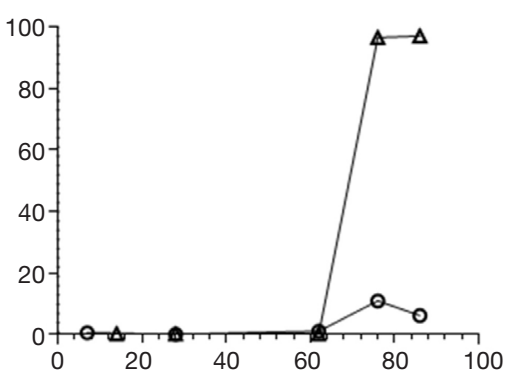

M7

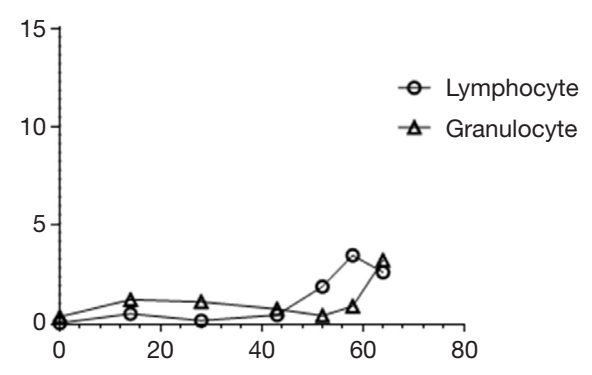

Post Operative Day

Figure 7 Flow cytometry plots of chimerism analysis of Group 2 recipients. Large spike in donor cells within the granulocyte population of M6 whole blood post-DBMT; donor cells in this gate are negative for almost all lineage markers. Very low percentage of donor cells was detected for M5 and M7 throughout the study. DBMT, delayed bone marrow transplantation.

premise of topical and/or local immunosuppressive therapy (i.e., to prevent the onset of Grade I-II rejection episodes). While Fries' results are encouraging with the use of the FK506-loaded hydrogels alone, our study represents an alternative approach towards FK506 treatment. We utilized FK506 implants, standard induction and maintenance triple immunosuppression concurrently (in Group 2), and provided proof-of-concept of the efficacy of our technology in mitigating acute rejection across the highest $\mathrm{MHC}$ mismatch barrier (i.e., full) possible. Additionally, 2 of 3 NHPs in Group 2 of the current study also demonstrated rejection-free VCA survival without additional IM FK506 dosing for 45-60 days (Figure 4).

This study is a proof-of-concept for clinical application of local FK506 implant technology. Additionally, it may prevent cases of VCA rejection that are due to patient noncompliance with oral immunosuppression. We do acknowledge that the current formulation, despite our best efforts, can lead to unacceptably high levels of systemic FK506. However, the surgically accessible subcutaneous position permits removal of some, or all of the implants in the event of unacceptably high levels.

Interestingly, high-dose FK506 has been explored as part of induction therapy prior to VCA in both NHP (29) and swine (30) models with varying results. In NHPs, high dose FK506 (systemic levels of 30-50 ng/mL) was maintained for 28 days before maintenance on once-daily IM dosing (target trough levels of 10-20 ng/mL) and 5 of 6 NHPs demonstrated clinically rejection-free (but histology revealed mild rejection) survival of up to 177 days (mean, 113 days). All five NHPs subsequently developed PTLD and no evidence of chimerism was detected throughout the study. In swine, full MHC-mismatch transplant pairs underwent VCA following conditioning with 50 cGy total body and 350 cGy thymic irradiation (i.e., a "day 0 " protocol rather than a DTIP) and results were highly variable. High dose FK506 (15-20 ng/mL) maintenance prevented VCA rejection in swine (3 of 3) but all developed major infectious complications leading to death. Swine that only received transient high dose FK506 for 60 days (3 of 3) had indefinite VCA survival ( $>300$ days) while off all immunosuppression without any mention of the presence or absence of chimerism.

Previous SOT studies in swine utilizing high-dose FK506 as induction over 12 days (31-33) have led to immune tolerance, so a species-specific mechanism may explain the differences in NHP versus swine experiments. Also, we have previously shown in swine VCA studies that transient mixed chimerism was insufficient for tolerance (34) and that stable mixed chimerism would be required instead (35), but this was dependent on the MHC barriers involved (36). Building on these mechanistic studies in swine, our laboratory has been focused on developing a successful mixed chimerism protocol in NHPs. Our recent work showed that DTIP, in which animals are kept on immunosuppression until healed and recovered from the initial tissue transplant procedure, then conditioned and given stem cells for tolerance induction months later, while successful in SOT (37), was not able to mitigate acute VCA rejection during the delay period. The rejection episodes negated development of mixed chimerism in NHPs (10), similar to the experience in clinical renal tolerance trials (38). While recent clinical developments have shown the intriguing promise of adding rituximab as part of induction to abrogate rejection episodes 

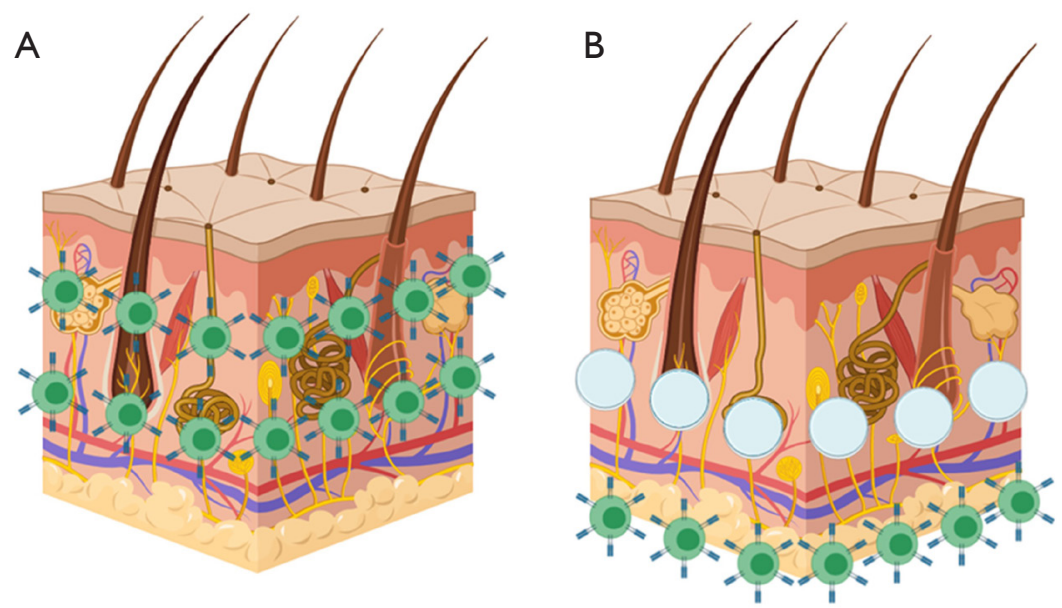

FK506 implants

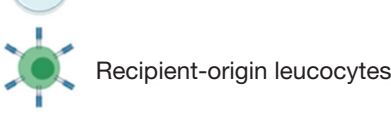

Figure 8 Proposed mechanism of action of local FK506 implant technology in averting rejection in vascularized composite allografts (VCA). (A) Previous studies from our laboratory suggest that skin cells within VCA dermis undergoes near complete turn-over and becomes infiltrated by recipient-origin leukocytes which likely results in rejection. (B) By delivering FK506 into the dermis directly through subcutaneous implantation, this infiltration of recipient-origin leukocytes may be delayed and/or negated completely, to mitigate the development of acute VCA rejection.

for up to two years post-VCA (39), the need for strict patient compliance with maintenance immunosuppression remains and demonstrates the potential utility of our local FK506 implants (Figure 8). Most encouragingly, there is now emerging evidence to support the use of rituximab prophylactically against PTLD (40), which is a strategy under consideration in our laboratory.

In conclusion, the recent development of DDS has shown much promise in controlling acute rejection of VCA. Our current study has shown that such proof-of-concept work in small animals can be scaled up into a clinically relevant large animal model (NHP). Future efforts will be focused on optimizing our local FK506 implant technology to minimize systemic morbidity by reducing the initial peak of burst release, in pursuance of the DTIP strategy for mixed chimerism and immune tolerance to VCA.

\section{Acknowledgments}

Presented (in part) at: the annual meetings of the Society for Biomaterials in Minneapolis, MN, 5-8 April 2017; Atlanta, GA, 11-14 April 2018; and Seattle, WA, 3-6 April 2019; the $14^{\text {th }}$ Congress of the International Society of
Vascularized Composite Allotransplantation, New Delhi, India, 1 October 2019 (The Transplantation Society Scientific Award Winner); and the $65^{\text {th }}$ Annual Meeting (virtual) of the Plastic Surgery Research Council, May 28-31, 2020.

Funding: This work was supported by the Office of the Assistant Secretary of Defense for Health Affairs and the Defense Health Agency, Research, Development and Acquisition Directorate through the Reconstructive Transplant Research Consortium under Award No. (W81XWH-15-1-0281). Opinions, interpretations, conclusions and recommendations are those of the authors and are not necessarily endorsed by the Department of Defense.

\section{Footnote}

Reporting Checklist: The authors have completed the ARRIVE reporting checklist. Available at http://dx.doi. org/10.21037/atm-21-313

Data Sharing Statement: Available at http://dx.doi. org/10.21037/atm-21-313

Peer Review File: Available at http://dx.doi.org/10.21037/ 
atm-21-313

Conflicts of Interest: All authors have completed the ICMJE uniform disclosure form (available at http://dx.doi. org/10.21037/atm-21-313). The authors have no conflicts of interest to declare.

Etbical Statement: The authors are accountable for all aspects of the work in ensuring that questions related to the accuracy or integrity of any part of the work are appropriately investigated and resolved. Experiments were performed under a project license (2015N000146) granted by the IACUC of Massachusetts General Hospital, in compliance with the NIH's Guide for the Care and Use of Laboratory Animals.

Open Access Statement: This is an Open Access article distributed in accordance with the Creative Commons Attribution-NonCommercial-NoDerivs 4.0 International License (CC BY-NC-ND 4.0), which permits the noncommercial replication and distribution of the article with the strict proviso that no changes or edits are made and the original work is properly cited (including links to both the formal publication through the relevant DOI and the license). See: https://creativecommons.org/licenses/by-nc-nd/4.0/.

\section{References}

1. Dubernard JM, Owen E, Herzberg G, et al. Human hand allograft: report on first 6 months. Lancet 1999;353:1315-20.

2. Petruzzo P SC, Lanzetta M, Dubernard JM. Report (2017) of the International Registry on Hand and Composite Tissue Allotransplantation (IRHCTT). Curr Transplant Reports 2017;4:294-303.

3. Petruzzo P, Dubernard JM. The International Registry on Hand and Composite Tissue allotransplantation. Clin Transpl 2011:247-53.

4. Petruzzo P, Lanzetta M, Dubernard JM, et al. The International Registry on Hand and Composite Tissue Transplantation. Transplantation 2010;90:1590-4.

5. Lee WP, Yaremchuk MJ, Pan YC, et al. Relative antigenicity of components of a vascularized limb allograft. Plast Reconstr Surg 1991;87:401-11.

6. Kueckelhaus M, Fischer S, Seyda M, et al. Vascularized composite allotransplantation: current standards and novel approaches to prevent acute rejection and chronic allograft deterioration. Transpl Int 2016;29:655-62.

7. Diaz-Siso JR, Fischer S, Sisk GC, et al. Initial experience of dual maintenance immunosuppression with steroid withdrawal in vascular composite tissue allotransplantation. Am J Transplant 2015;15:1421-31.

8. Chen YT, Huang SC, Chen CC, et al. Topical tacrolimus and steroids modulate $\mathrm{T}$ cells in acute rejection of hand allotransplantation: Two case reports. Microsurgery 2020;40:217-23.

9. Gama AR, Ng ZY, Shanmugarajah K, et al. Local Immunosuppression for Vascularized Composite Allografts: Application of Topical FK506-TyroSpheres in a Nonhuman Primate Model. J Burn Care Res 2020;41:1172-8.

10. Lellouch AG, Ng ZY, Rosales IA, et al. Toward Development of the Delayed Tolerance Induction Protocol for Vascularized Composite Allografts in Nonhuman Primates. Plast Reconstr Surg 2020;145:757e-68e.

11. Sykes M. Mixed chimerism and transplant tolerance. Immunity 2001;14:417-24.

12. Kawai T, Sachs DH, Sykes M, et al. HLAmismatched renal transplantation without maintenance immunosuppression. N Engl J Med 2013;368:1850-2.

13. Taddeo A, Tsai C, Vogelin E, et al. Novel targeted drug delivery systems to minimize systemic immunosuppression in vascularized composite allotransplantation. Curr Opin Organ Transplant 2018;23:568-76.

14. Dhayani A, Kalita S, Mahato M, et al. Biomaterials for topical and transdermal drug delivery in reconstructive transplantation. Nanomedicine (Lond) 2019;14:2713-33.

15. Leonard DA, Gordon CR, Sachs DH, et al. Immunobiology of face transplantation. J Craniofac Surg 2012;23:268-71.

16. Fries CA, Lawson SD, Wang LC, et al. Graft-implanted, enzyme responsive, tacrolimus-eluting hydrogel enables long-term survival of orthotopic porcine limb vascularized composite allografts: A proof of concept study. PLoS One 2019;14:e0210914.

17. O'Connor SL, Blasky AJ, Pendley CJ, et al. Comprehensive characterization of MHC class II haplotypes in Mauritian cynomolgus macaques. Immunogenetics 2007;59:449-62.

18. Pendley CJ, Becker EA, Karl JA, et al. MHC class I characterization of Indonesian cynomolgus macaques. Immunogenetics 2008;60:339-51.

19. Barth RN, Bluebond-Langner R, Nam A, et al. Facial subunit composite tissue allografts in nonhuman primates: I. Technical and immunosuppressive requirements for prolonged graft survival. Plast Reconstr Surg 2009;123:493-501.

20. Molde Joseph MA, Steele Joseph AM, Lima Mariana RN, et al. Localized tacrolimus delivery for transplant rejection 
prevention via tyrosphere-loaded rapidly soluble films. Transactions of the Annual Meeting of the Society for Biomaterials and the Annual International Biomaterials Symposium April 2019; Volume 40:5104.

21. Cendales LC, Kanitakis J, Schneeberger S, et al. The Banff 2007 working classification of skin-containing composite tissue allograft pathology. Am J Transplant 2008;8:1396-400.

22. Schneider M, Cardones AR, Selim MA, et al. Vascularized composite allotransplantation: a closer look at the banff working classification. Transpl Int 2016;29:663-71.

23. Boskovic S, Kawai T, Smith RN, et al. Monitoring antidonor alloantibodies as a predictive assay for renal allograft tolerance/long-term observations in nonhuman primates. Transplantation 2006;82:819-25.

24. Kawai T, Cosimi AB, Sachs DH. Preclinical and clinical studies on the induction of renal allograft tolerance through transient mixed chimerism. Curr Opin Organ Transplant 2011;16:366-71.

25. Solari MG, Washington KM, Sacks JM, et al. Daily topical tacrolimus therapy prevents skin rejection in a rodent hind limb allograft model. Plast Reconstr Surg 2009; 123:17S-25S.

26. Gharb BB, Rampazzo A, Altuntas SH, et al. Effectiveness of topical immunosuppressants in prevention and treatment of rejection in face allotransplantation. Transplantation 2013;95:1197-203.

27. Gajanayake T, Olariu R, Leclere FM, et al. A single localized dose of enzyme-responsive hydrogel improves long-term survival of a vascularized composite allograft. Sci Transl Med 2014;6:249ra110.

28. Feturi FG, Wang H, Brudno Y, et al. Abstract 23: Ultrasound-mediated on-demand release from ionically cross-linked hydrogel: New approach for targeted Immunotherapy in Vascularized Composite Allotransplantation. Plast Reconstr Surg Glob Open 2017;5:18-9.

29. Unadkat JV, Schnider JT, Feturi FG, et al. Single Implantable FK506 Disk Prevents Rejection in Vascularized Composite Allotransplantation. Plast Reconstr Surg 2017;139:403e-14e.

30. Sutter D, Dzhonova DV, Prost JC, et al. Delivery of Rapamycin Using In Situ Forming Implants Promotes Immunoregulation and Vascularized Composite Allograft Survival. Sci Rep 2019;9:9269.

31. Barth RN, Nam AJ, Stanwix MG, et al. Prolonged survival of composite facial allografts in non-human primates associated with posttransplant lymphoproliferative disorder. Transplantation 2009;88:1242-50.

32. Leto Barone AA WH, Swanson EW, et al. Abstract 45: Vascularized Composite Allograft Tolerance Across A Full MHC Mismatch Is Possible With Transient High Dose Tacrolimus. Plast Reconstr Surg - Glob Open 2017;5:34-5.

33. Madariaga ML, Michel SG, Tasaki M, et al. Induction of cardiac allograft tolerance across a full MHC barrier in miniature swine by donor kidney cotransplantation. Am J Transplant 2013;13:2558-66.

34. Madariaga ML, Spencer PJ, Michel SG, et al. Effects of Lung Cotransplantation on Cardiac Allograft Tolerance Across a Full Major Histocompatibility Complex Barrier in Miniature Swine. Am J Transplant 2016;16:979-86.

35. Utsugi R, Barth RN, Lee RS, et al. Induction of transplantation tolerance with a short course of tacrolimus (FK506): I. Rapid and stable tolerance to two-haplotype fully mhc-mismatched kidney allografts in miniature swine. Transplantation 2001;71:1368-79.

36. Leto Barone AA KJ, Albritton A, et al. Effects of Transient Donor Chimerism on Rejection of MHC-Mismatched Vascularized Composite Allografts in Swine. Vasc Compos Allotransplantation 2015;2:1-8.

37. Leonard DA, Kurtz JM, Mallard C, et al. Vascularized composite allograft tolerance across MHC barriers in a large animal model. Am J Transplant 2014;14:343-55.

38. Shanmugarajah K, Powell H, Leonard DA, et al. The Effect of MHC Antigen Matching Between Donors and Recipients on Skin Tolerance of Vascularized Composite Allografts. Am J Transplant 2017;17:1729-41.

39. Gelb BE, Diaz-Siso JR, Plana NM, et al. Absence of Rejection in a Facial Allograft Recipient with a Positive Flow Crossmatch 24 Months after Induction with Rabbit Anti-Thymocyte Globulin and Anti-CD20 Monoclonal Antibody. Case Rep Transplant 2018;2018:7691072.

40. Lindsay J, Yong MK, Greenwood M, et al. Epstein-Barr virus related post-transplant lymphoproliferative disorder prevention strategies in allogeneic hematopoietic stem cell transplantation. Rev Med Virol 2020;30:e2108.

Cite this article as: Lellouch AG, Taveau CB, Andrews AR, Molde J, Ng ZY, Tratnig-Frankl P, Rosales IA, Goutard M, Lupon E, Lantieri LA, Colvin RB, Randolph MA, Kohn J, Cetrulo CL Jr. Local FK506 implants in non-human primates to prevent early acute rejection in vascularized composite allografts. Ann Transl Med 2021;9(13):1070. doi: 10.21037/ atm-21-313 